\title{
美捷登年终盘点：2020年 十大科学事件
}

裴磊

华中科技大学同济医学院

2020 年全世界遭遇前所未有的挑战, 不管今天 是哪天、哪周或是哪月, 全世界的科学家们一直在 不解努力去发现和创新。这是对科学技术在社会中 的重要性证明, 也是对化学家、数学家、物理学家、
生物学家、生态学家等等等和其他许多自然与社 会科学家所思所想的证明。正是因为这些科学家, 使得世界不断向前发展。诚然, 今年是灾难性的一年, 在即将结束之际, 让我们花点时间来盘点一下今年

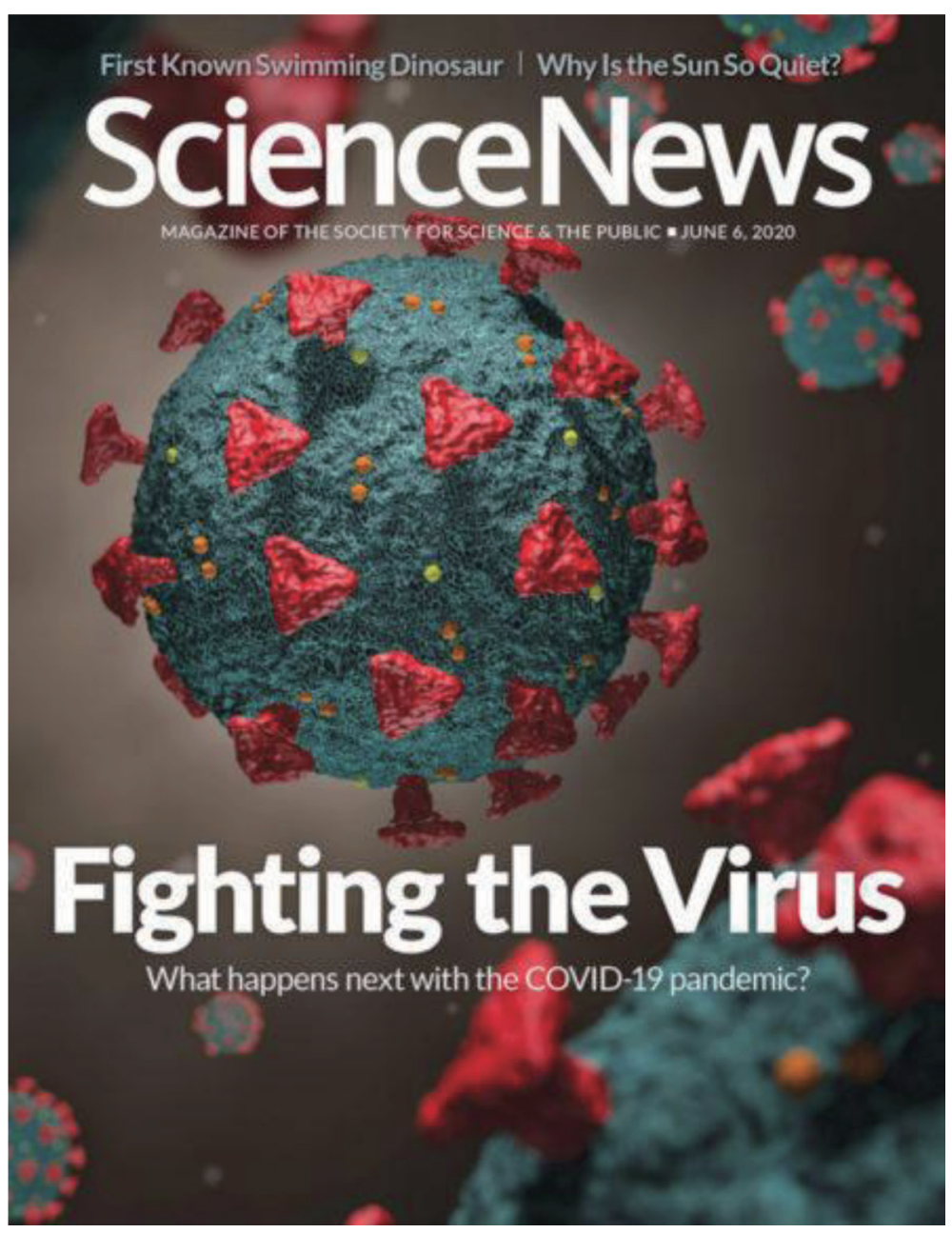

图片来源：June 6, 2020 | Science News. 


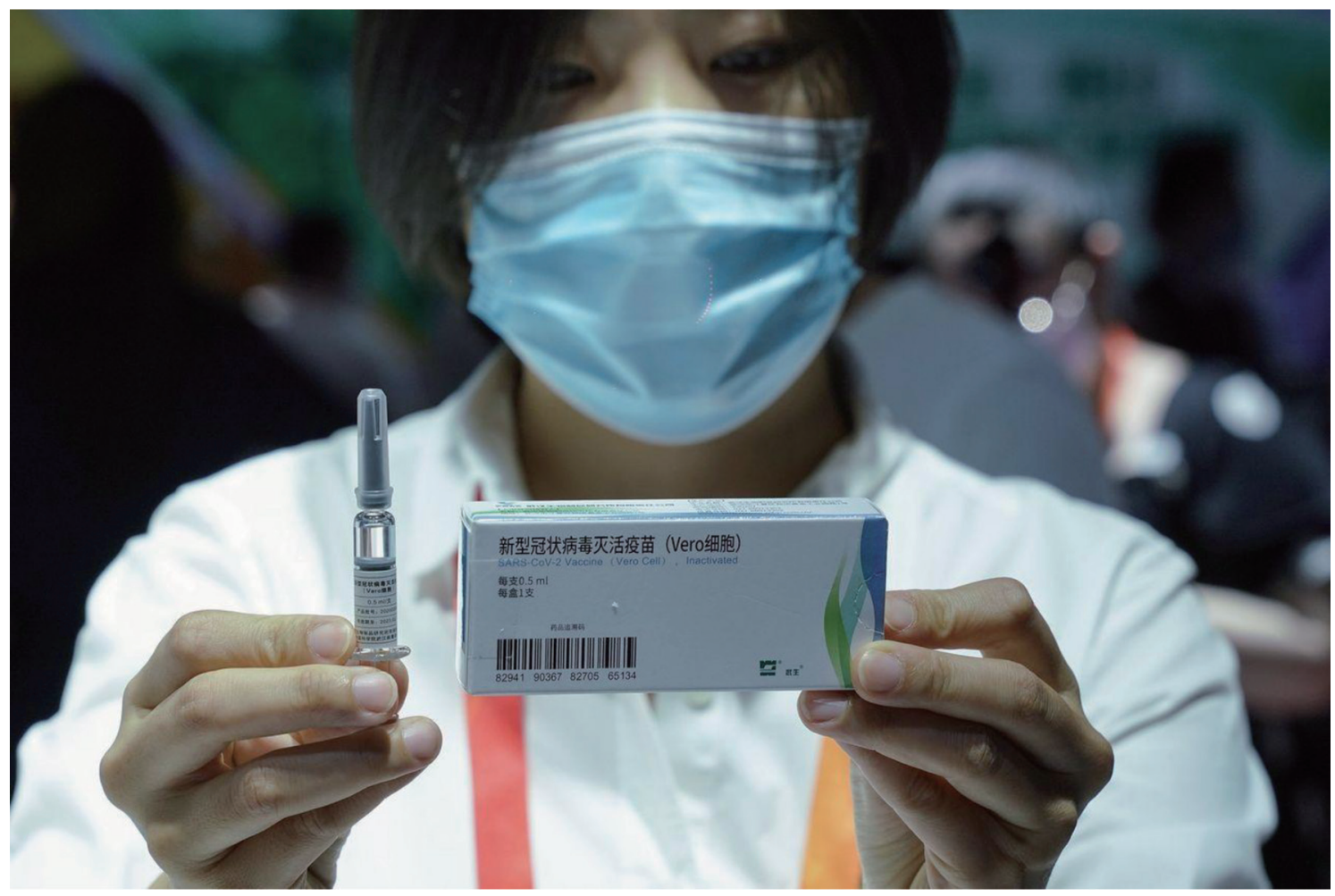

图1 中国生物新型冠状病毒灭活疫苗。

图片来源: 中国已为数十万人接种试验性新冠病毒疫苗 - 华尔街日报(wsj.com)

在科学领域值得让我们兴奋和记住的一些事情。

\section{SARS-CoV-2与COVID-19疫苗研究}

中国政府和人民付出巨大努力短时间内控制了 新冠病毒蔓延, 为全球树立了榜样。同时, 中国科 学家在研究新型冠状病毒和制止全球大流行方面 所取得的研究和进展是前所未有的。《实验室设备 COVID-19 资源指南》按时间顺序汇编了我们今年 的重点报道的 COVID-19 文章。这份清单是科学方 法的直观展示, 从早期羟基氯喹被吹捧到不再作为 治疗手段, 从缺乏个人防护装备到 $3 \mathrm{~d}$ 打印口罩, 从 第一阶段疫苗试验到 $95 \%$ 的有效性。科学家们全力 以赴, 找出哪些治疗会起作用, 哪些不会。现在, 多亏了这些科学家, 我们终于看到了一线曙光。

我国目前已有 5 个新冠病毒疫苗进行III期临床 试验, 数量位于全球前列。疫苗研发已经进入冲刺 阶段, 我们处于全球第一方阵, 但不为第一而抢跑。 已经进入III期临床试验的疫苗包括国药集团中国生 物的 2 个灭活疫苗 (图 1)、北京科兴中维公司的 1
个灭活疫苗、军事医学研究院和康希诺公司联合研 发的腺病毒载体疫苗、中科院微生物所和智飞生物 公司联合研发的重组蛋白疫苗。由于新冠肺炎疫情 在我国得到有效控制, 国内已不具备开展III期临床 试验的条件, 因此上述III期临床试验主要在海外进 行, 也面临着一些困难和挑战。FDA 的疫苗及相关 生物制品咨询委员会近期以 17 票对 4 票, 1 票弃权 通过了辉瑞 COVID-19 疫苗 BNT162b2 的紧急使用 授权。FDA 通知辉瑞和 BioNTech, 将 “迅速完成 并发布紧急使用授权”。美国的 COVID-19 疫苗接 种很快可以开始, FDA 也表示已经通知了 CDC 和 Warp Speed 运营部，以便他们可以据此计划分发。

\section{2. 人工智能几乎完美地识别出乳腺癌和前列 腺癌}

今年 1 月, 研究人员发表的研究结果显示, 谷 歌公司的 DeepMind 人工智能系统在发现近 2.9 万名 女性的 $\mathrm{x}$ 射线图像异常方面优于 6 名人类放射科医 生。在这项研究中, 人工智能系统显示误报率降低了 


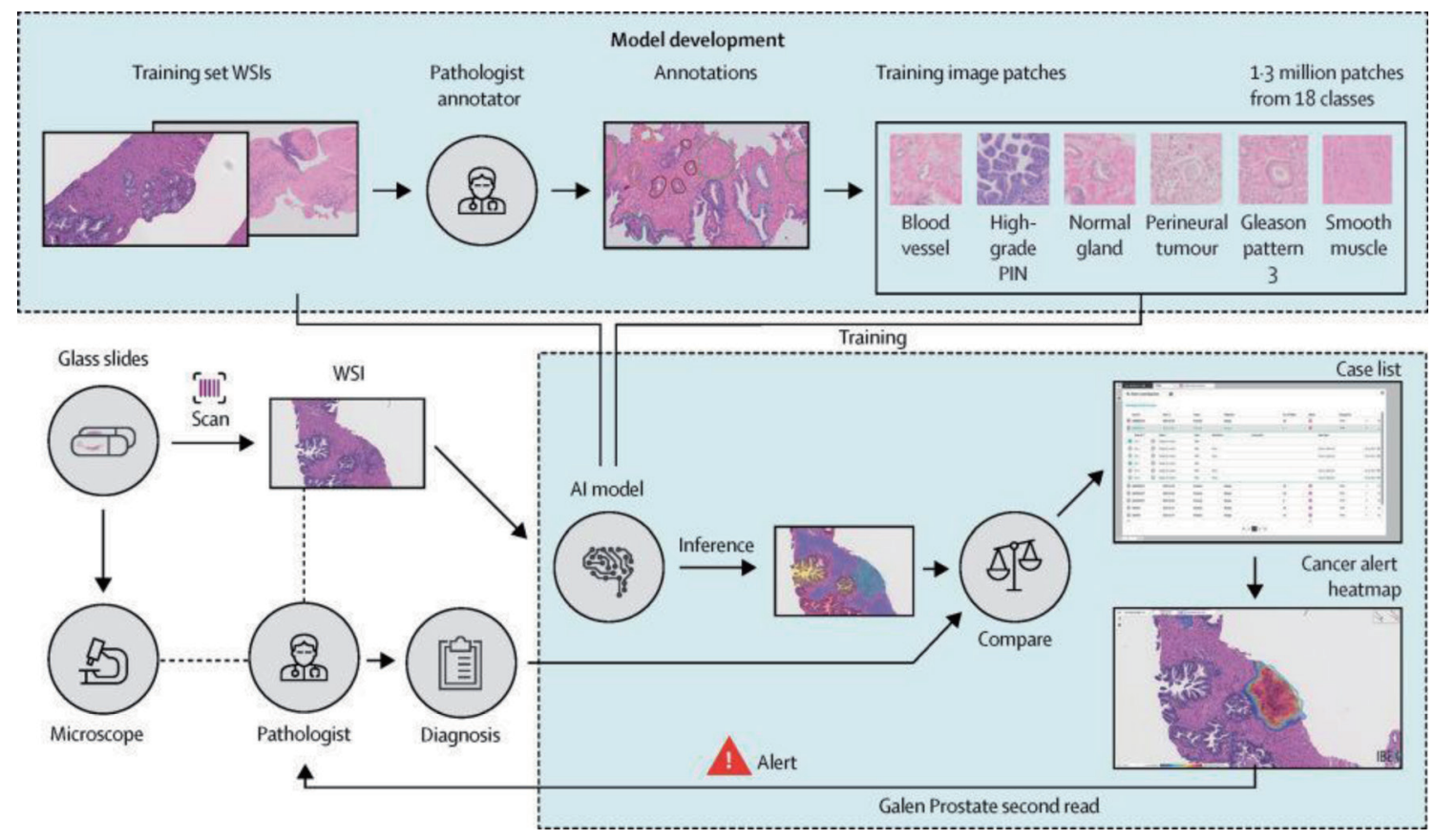

图2 前列腺癌人工智能诊断核心穿刺活检全切片图像算法: 盲法临床验证和应用研究。

图片来自An artificial intelligence algorithm for prostate cancer diagnosis in whole slide images of core needle biopsies: a blinded clinical validation and deployment study - The Lancet Digital Health

$5.7 \%$ 。在今年 7 月进行的另一项研究中, 匹兹堡大学 (University of Pittsburgh) 的研究人员训练了一个人工 智能程序, 可以通过组织切片识别前列腺癌 (图 2)。 在检测过程中, 人工智能检测前列腺癌的敏感性为 98\%，特异性为 $97 \% ，$ 显著高于此前报道的水平。该 人工智能系统是第一个扩展到癌症检测之外的系统, 报告肿瘤分级、大小和周围神经侵犯的高性能, 所有 临床重要特征都是典型病理报告的一部分。

\section{CRISPR-Cas9首次在人体内使用}

今年 3 月, 作为 BRILLANCE 临床试验的一部 分, 俄勒冈健康与科学大学 (Oregon Health \& Science University) 的医生进行了首次体内 CRISPR 基 因编辑程序 (图 3)，以解决导致失明的基因突变问 题。参与试验的患者患有利伯尔先天性黑朦, 这种 黑朦是由一种基因突变导致的，这种基因突变使身 体无法产生一种将光转化为信号所需的蛋白质。在 这种情况下, 科学家们通过在突变基因的两侧进行 两次切割来编辑 / 删除突变, 依靠 DNA 的末端重 新连接, 让基因按照应有的方式工作。在早期对人 体组织、小鼠和猴子进行的测试中, 科学家们能够
纠正一半带有致病突变的细胞, 这超过了恢复视力 所需的量。

\section{4. 澳大利亚和美国加利福尼亚发生史无前 例的大火}

2019-2020 年澳大利亚森林火灾季节的一项研 究发现, 澳大利亚 $21 \%$ 的森林 (不包括塔斯马尼亚 岛) 被烧毁 (图 4), 这一数字被描述为前所未有的, “大大超过澳大利亚和全球” 过去 20 年以前的火灾。 这篇发表在《自然气候变化》杂志上的一篇研究报 告的社论写道, 异乎寻常的大范围火灾已经导致许 多人将澳大利亚称为气候变化的归零地, 这激发了全 世界要求澳大利亚和其他富裕国家肩负起保护环境 责任的呼声。

\section{5. 犬骨癌免疫治疗的成功进展到人类脑癌 试验}

今年 6 月, 密苏里大学 (University of Missouri) 的兽医研究人员利用个性化药物成功治疗了 14 只狗 


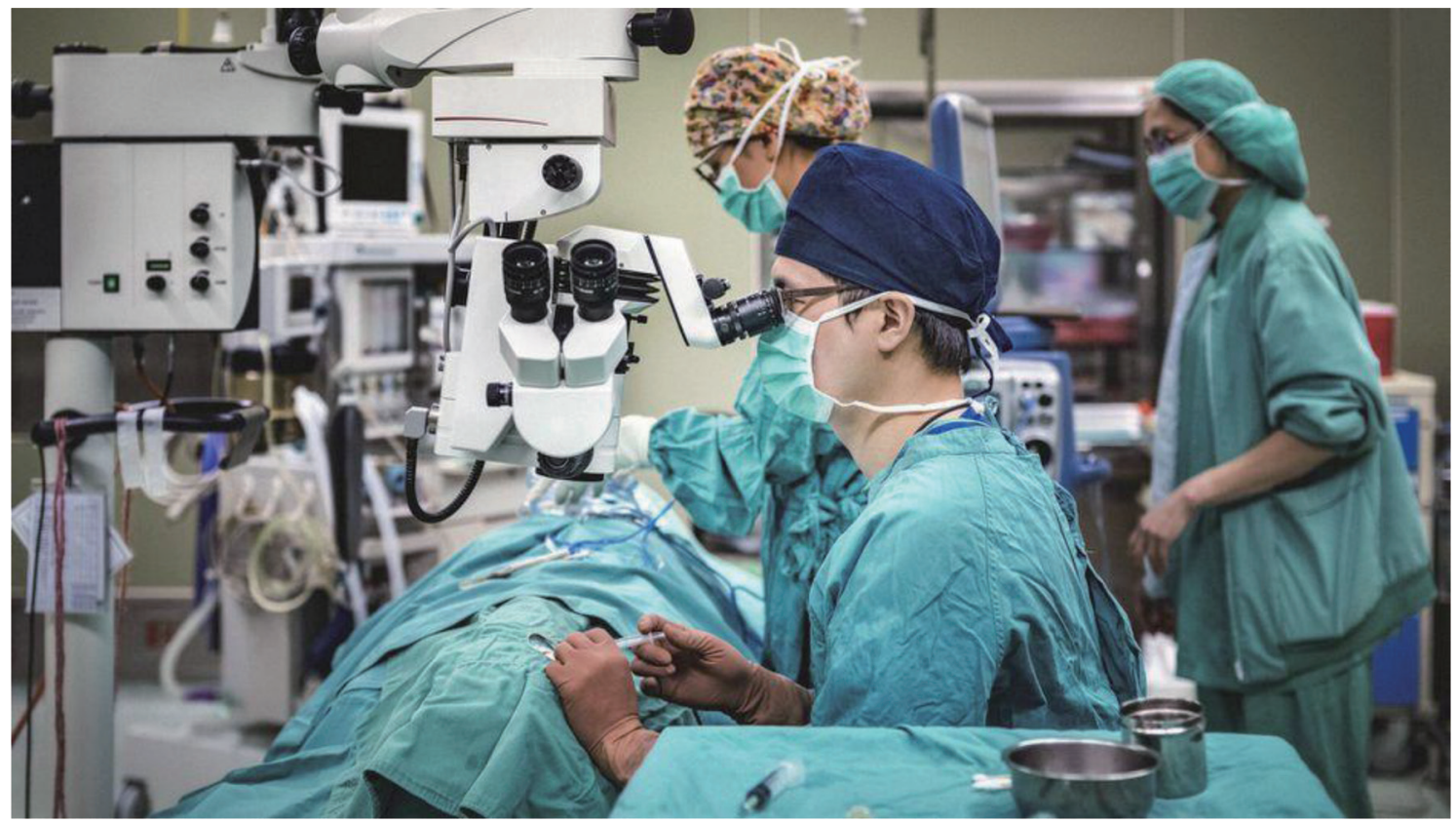

图3 CRISPR疗法治疗遗传性失明的首次临床试验。

图片来自CRISPR gene-editing tool used inside humans for the first time - CNET

的骨癌。通过从狗自身的肿瘤中创造出疫苗, 科学 家们能够瞄准特定的癌细胞, 避免化疗的毒副作用。 与截肢和化疗的平均生存时间相比, 接受免疫疗法 治疗的狗在开始治疗后多活了几个月, 有的超过两年。 这项研究的成功结果帮助研究小组获得了 FDA 的批 准，可以在人类脑癌患者身上测试这种方法。

\section{6. 商业太空飞行时代开始了}

去年 11 月, SpaceX 公司的 “龙的韧性” 号载人 飞船发射升空时 (图 6)，它所做的远不止将四名宇 航员送入国际空间站。此次发射标志着商业实体首 次成功地将美国宇航局宇航员送入太空中的高科技

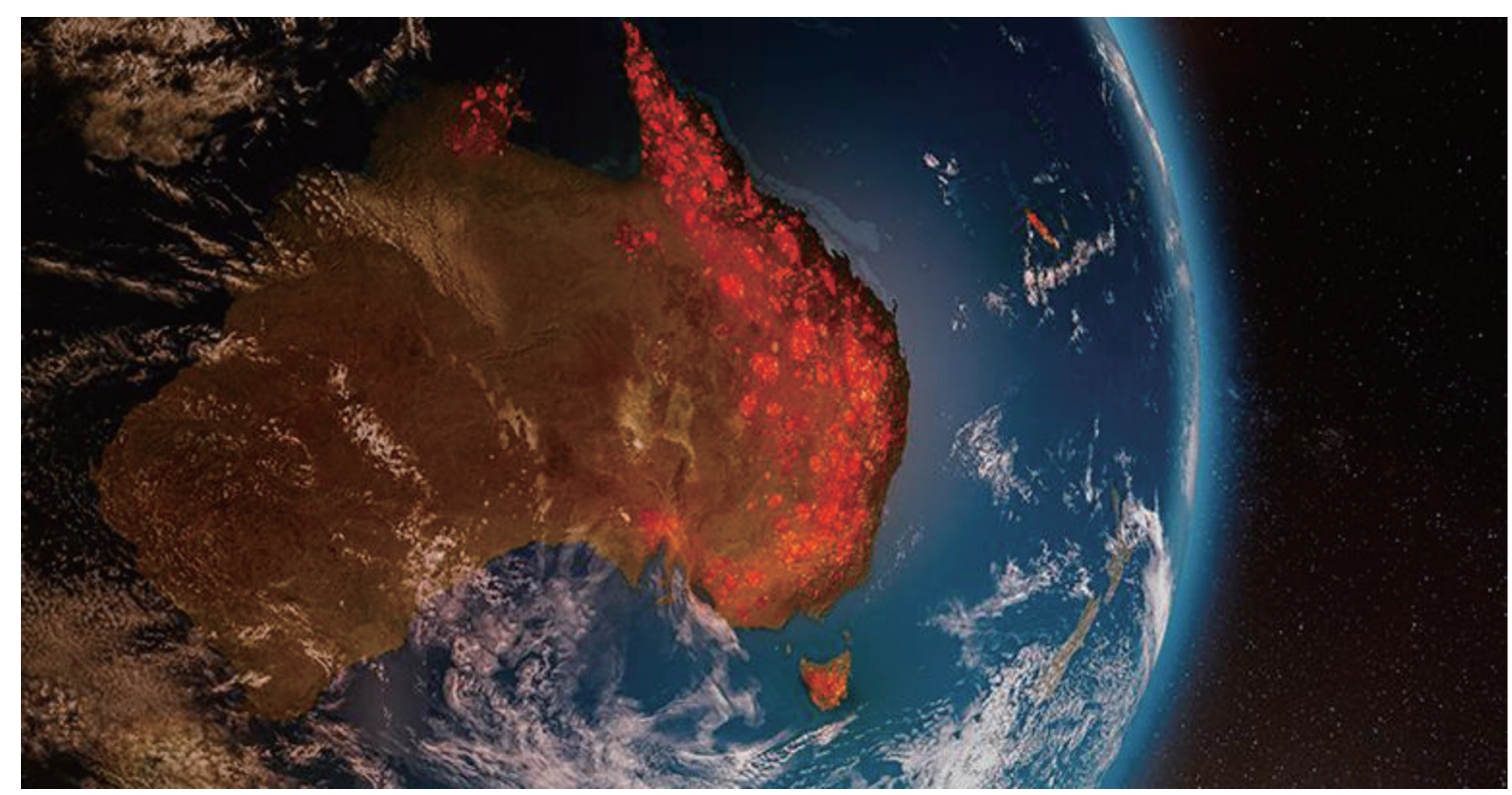

图4 澳洲火灾地图显示澳大利亚野火危机。

图片来自How are Aussie charities helping in the bushfire crisis? | PBA (probonoaustralia.com.au) 


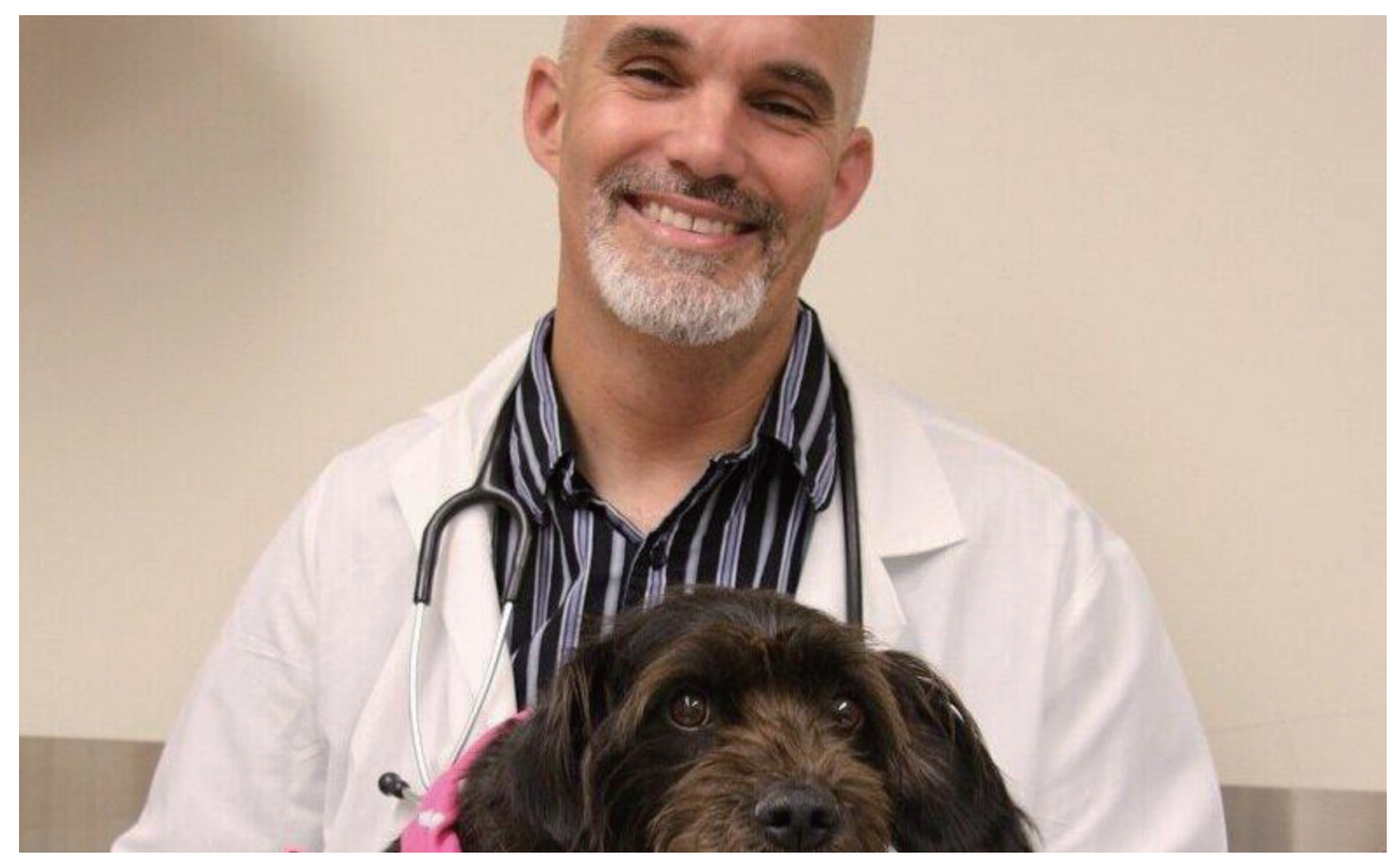

图5 用犬自身肿瘤制成的疫苗成功治疗犬骨癌。

图片来自Canine bone cancer successfully treated with vaccine made from dog's own tumor (phys.org)

实验室。几个月前, 太空计划又取得了另一个第一, 即最新的火星探测器 “毅力” 于 7 月发射时, 它成 为了第一个完全由美国制造的钚提供动力的探测器。 美国在 1988 年停止生产钚238。在那之后的几年
里, 美国动用了储备, 并从俄罗斯采购补充, 但这一 协议在 2010 年解除。因此, 2012 年, 奥巴马政府 和 NASA 与能源部达成协议, 利用 NASA 资金恢复 钚 238 的生产。“毅力” 项目的成功启动是这个每年

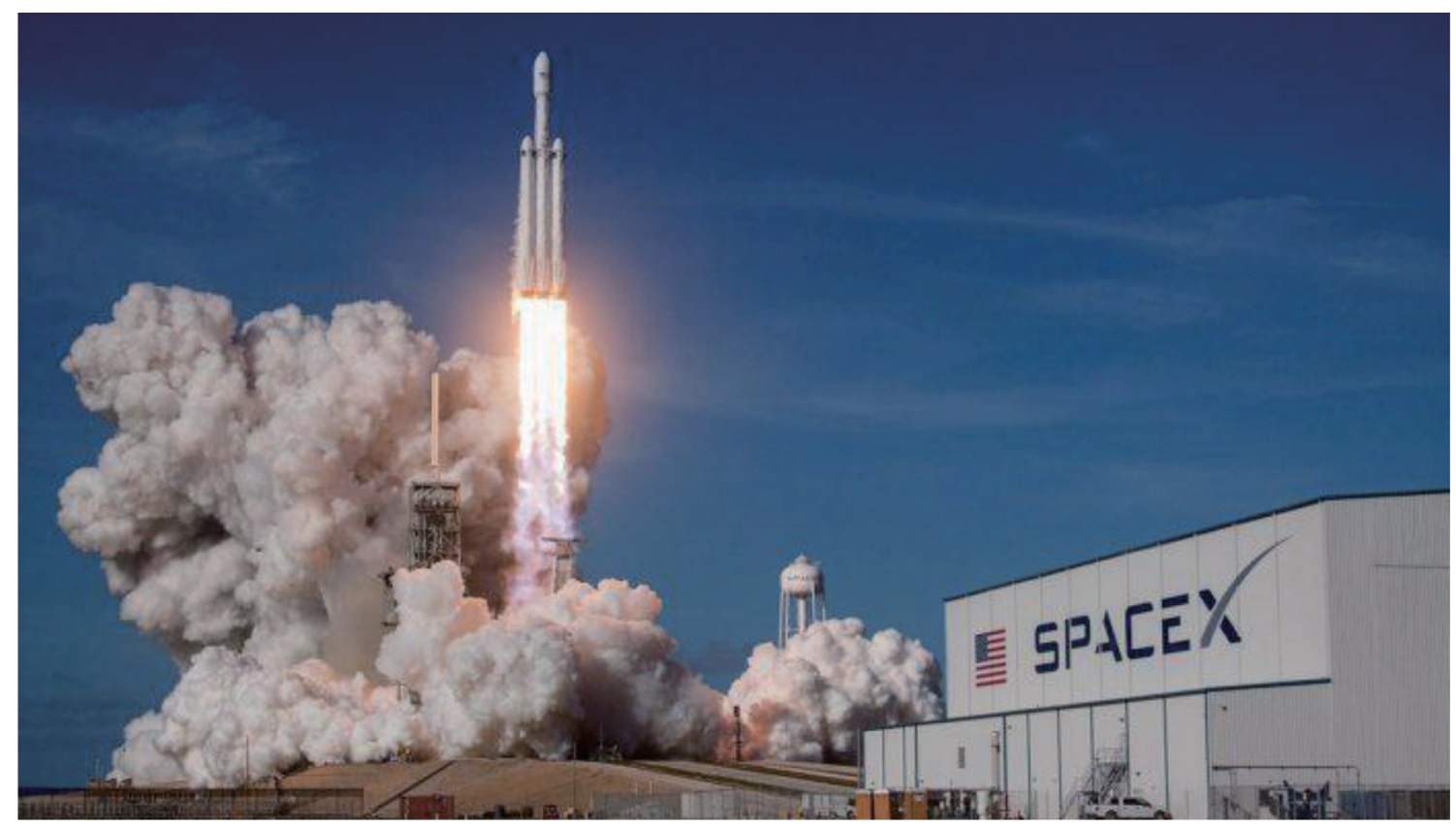

图6 商业太空旅行的黎明: SpaceX成功将2名美国宇航局宇航员送入轨道。

图片来自Dawn of commercial space travel: SpaceX successfully propels 2 NASA astronauts into orbit (theprint.in) 


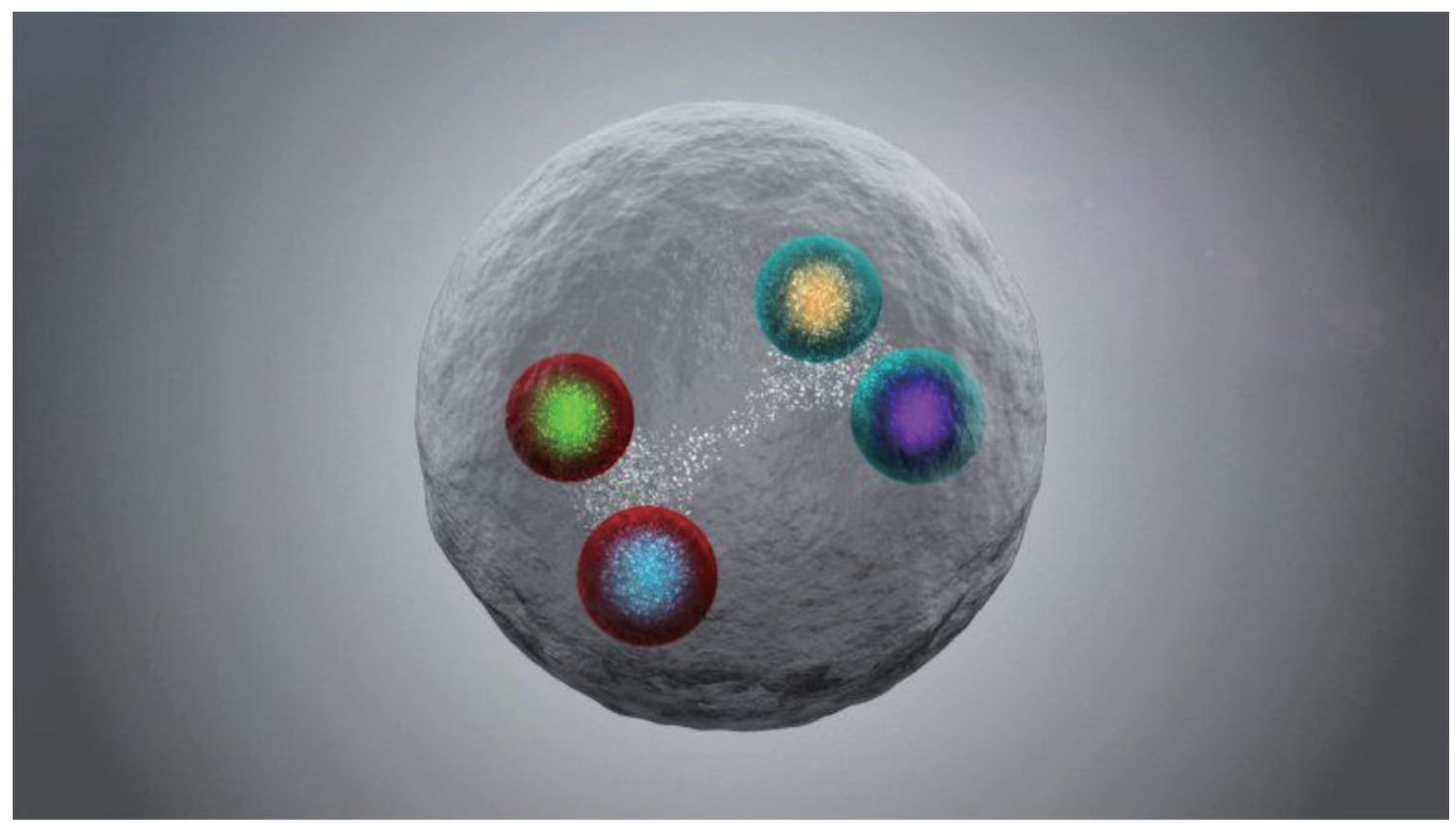

图7 用大型强子对撞机发现了从未见过的奇异四夸克粒子。

由两个魅惑夸克和两个魅惑反夸克组成的四夸克的插图, 首次被欧洲核子研究中心的LHCb合作所发现。来源: 欧洲核子 研究中心Exotic Never Before Seen Four-Quark Particle Discovered Using Large Hadron Collider (scitechdaily.com)

1500 万美元项目中最大的里程碑。

\section{7. 欧洲粒子物理研究所CERN发现了一种以 前从未见过的新型粒子}

在欧洲核子研究中心 $\mathrm{LHCb}$ 合作项目中工作的 科学家们发现了一种以前从未见过的四夸克粒子 (图 7)。这一发现很可能是之前未发现的一类粒子中的第 一个。这一发现将帮助物理学家更好地理解夸克结 合成复合粒子的复杂方式, 例如在原子核中发现的 无处不在的质子和中子。 $\mathrm{LHCb}$ 合作项目负责人乔瓦 尼・帕萨列娃 (Giovanni Passaleva) 说: “由四个夸克 组成的粒子已经很奇特了, 我们刚刚发现的这个粒子 是第一个由四个相同类型的重夸克组成的粒子, 特 别是两个魅惑夸克和两个魅惑反夸克”。

\section{8. 考古学家把美洲殖民的时间往前推了 15000年}

在墨西哥洞穴中发现的考古分析和对洞穴沉积 物的 DNA 分析揭示了一个不同的美洲殖民故事 (图 8)。有证据表明，人类在 3 万年前来到这个洞穴，
比预想的时间早了 1.5 万年。这一发现挑战了普遍持 有的理论，即克洛维斯人是 15000 年前美洲的第一 批人类居民。当著名的克洛维斯人进入美洲时, 早 期的美洲人在几千年前就已经消失了。墨西哥萨卡 特卡斯大学和埃克塞特大学的考古学家普利安 - 阿 德莱安说, 可能有很多殖民失败, 随着时间的流逝, 没有在今天的人群中留下遗传痕迹。

\section{9. 南极海冰上首次发现了微塑料污染恶化 的情况}

研究人员首次在南极海冰中发现了微塑料污染 (图 9)。这项研究由塔斯马尼亚大学的研究人员进行, 他们分析了 2009 年在南极洲东部收集的冰芯，发 现了来自 14 种不同类型聚合物的 96 个微塑料颗粒。 首席研究员安娜・凯利 (Anna Kelly) 表示, 南大洋 的偏远程度不足以保护它免受塑料污染的影响, 塑 料污染目前在全球各大洋都很普遍。澳大利亚研究 人员 10 月份的一项研究证实了凯利的想法。CSIRO 的研究人员记录到, 在首次全球海床上估计有 1400 万吨微塑料。这一数量是上次估计的海底数量的两 倍多。 


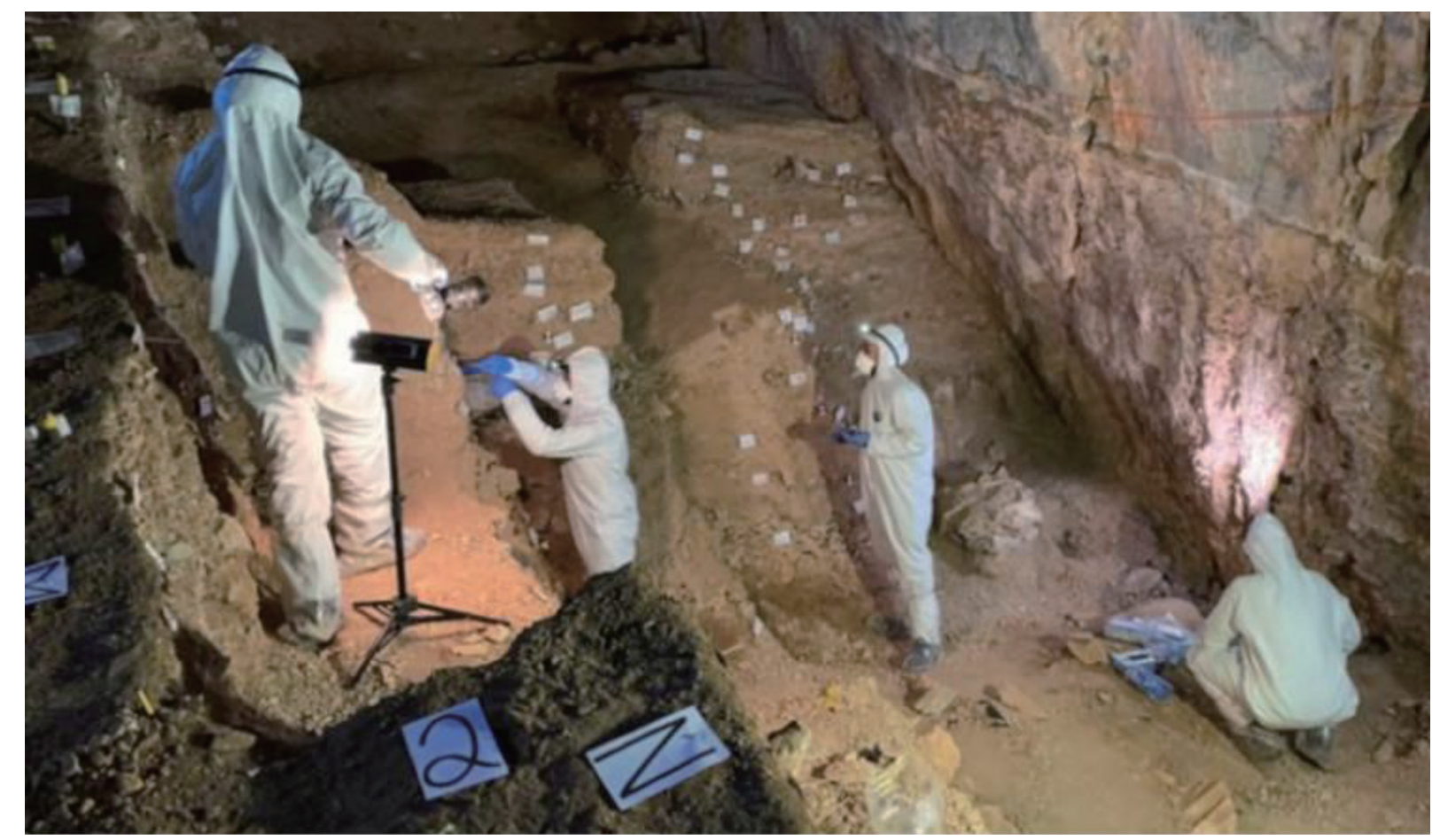

图8 Chiquihuite 洞穴中考察的科学家。

资料来源: Ancient Architects Humans Were in the Americas 15,000 Years Earlier Than Previously Thought (interestingengineering.com)

\section{WHERE DO MICROPLASTICS COME FROM?}

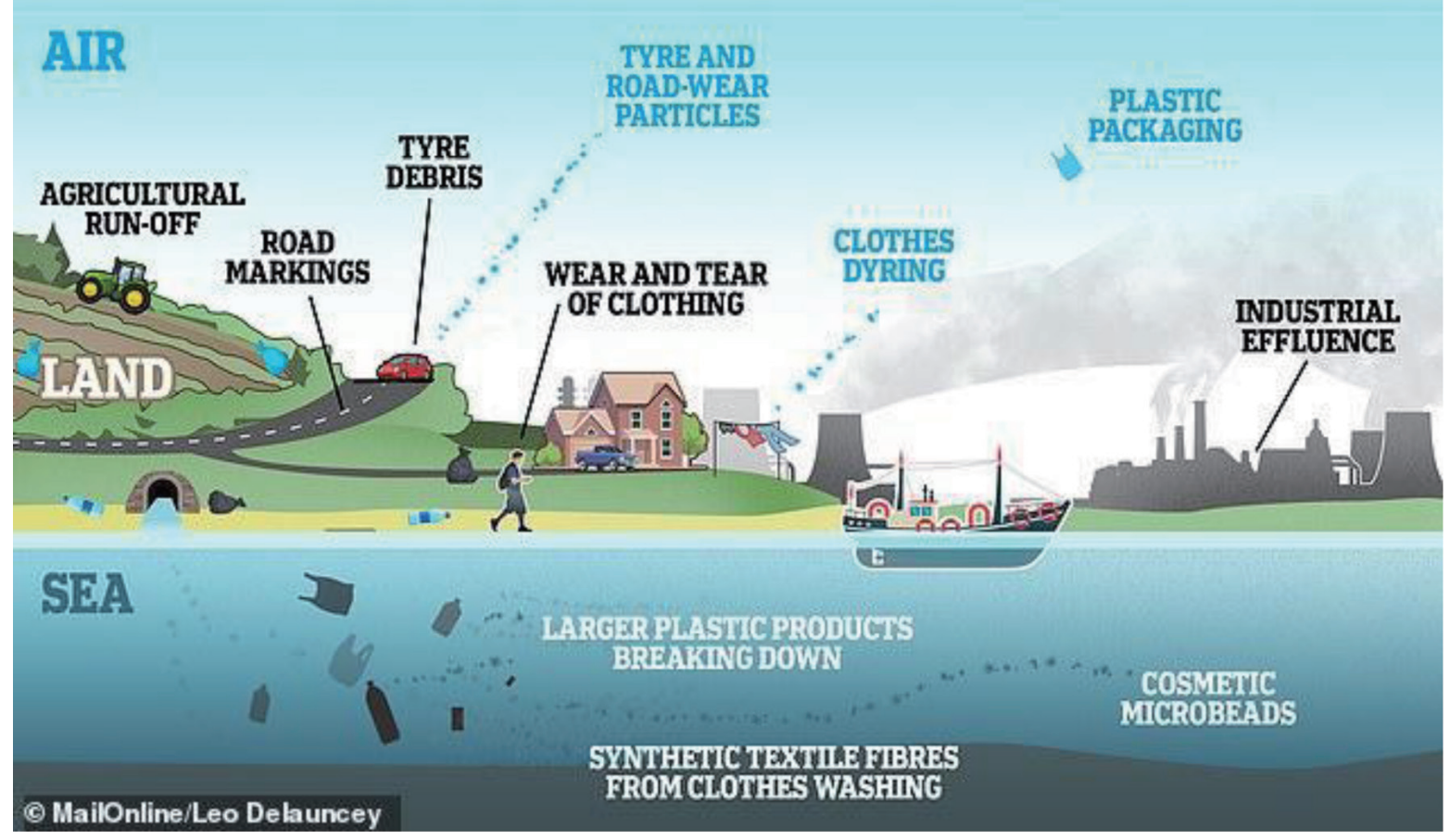

图9 微塑料通过各种方式进入水道, 最终悬浮在液体中。

它们可以通过水和空气进行长距离运输, 把它们带到世界上最遥远的角落。图片来源Micro-

plastics are found for the first time in Antarctic sea ice | Daily Mail Online 


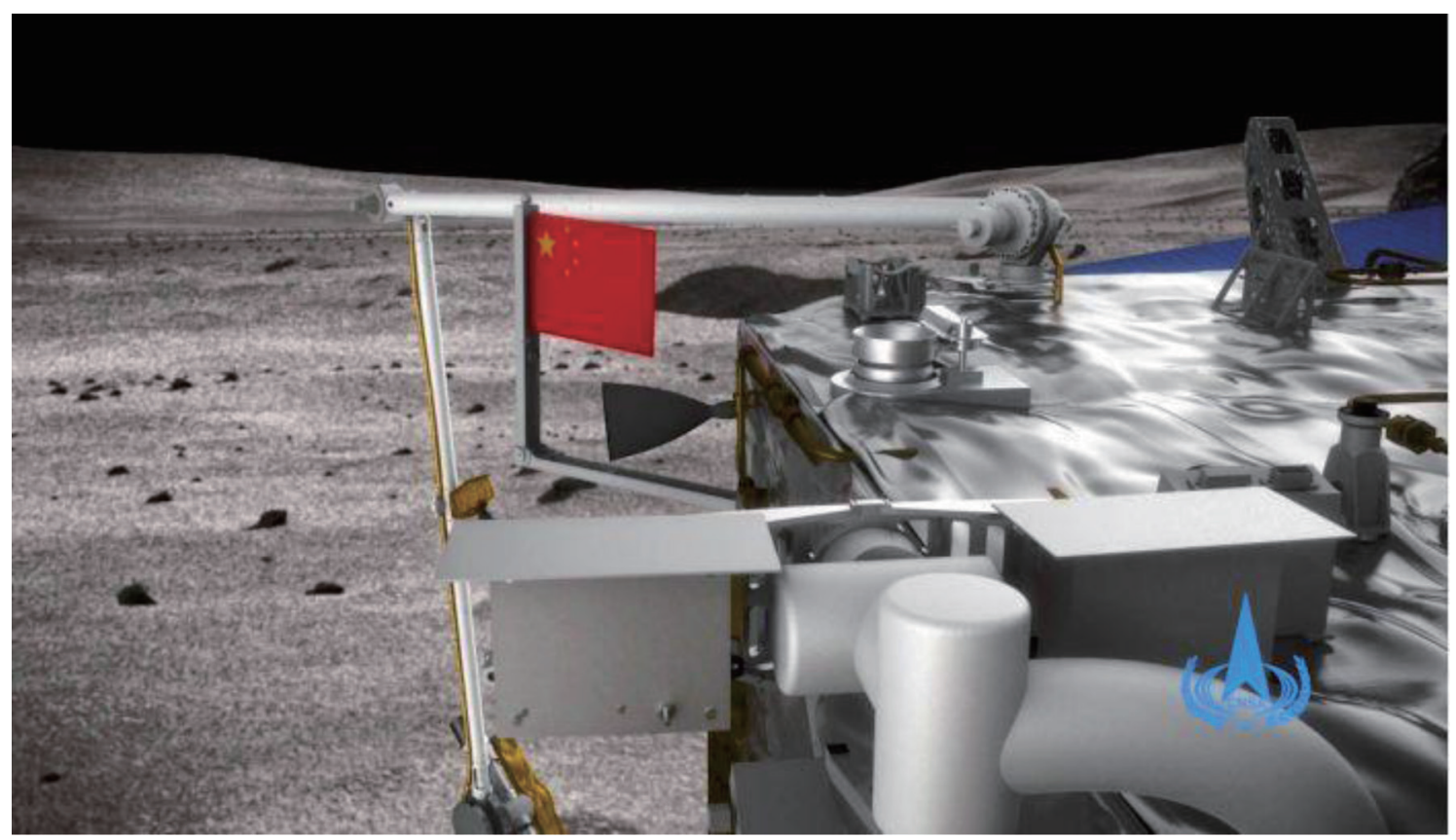

图10 中国首度在月球表面实现五星旗的独立展示（中国探月与深空探测网）。

图片来源https://www.worldjournal.com/wj/story/121474/5065419?from=wj_msg\&zh-cn

10. 中国嫦娥五号首次实现了中国地外天体 采样返回

中国探月工程嫦娥五号返回器携带月球样品, 于当地时间 17 日凌晨着陆地球, 嫦娥五号返回器 在内蒙古四子王旗预订区域成功着陆。这是中国首 次完成月球采样返回任务, 也是人类四十年来首次。 嫦娥五号于 12 月 1 日登陆月球表面 (图 10), 紧接 着开始采样, 两天后启动了重返地球旅程。中国科 学家希望通过采集月球样品, 更多地了解月球是如何 诞生的以及了解月球表面的火山形成和爆发周期。

嫦娥五号返回器成功返回地球后, 中国由此成 为继美国、前苏联分别于 1960 和 1970 年之后, 第 三个携带月球样品返回地球的国家。这也是自 1976 年苏联月球 24 号探月取样任务完成后的首次类似 的探月旅行。嫦娥五号的使命旨在月球上一直未有 探索过的区域采集两公斤的样品。嫦娥五号任务实 现了中国首次月面采样与封装、月面起飞、月球轨 道交会对接、携带样品再入返回等多项重大突破。
嫦娥五号携带月球样品成功返回, 这是中国航天计 划迈出的新的一步。2019 年年初, 中国发射的一 个探索器在月球背面降落, 为全球首创。中国希望 最终派遣宇航员登上月球。嫦娥五号首次实现了中 国地外天体采样返回, 标志着中国航天向前迈出的 一大步。

\section{结束语}

2020 年是一个大家都不愿回忆, 希望早点结束 的一年。尽管大多数人可能会将 2020 年与新冠肺炎 联系在一起, 但我们也应该看到一系列科学技术成 就。我们的盘点并不完全, 还有很多重要的发现并 未列入。不管怎样, 2020 年是太空探索、医学诊断、 神经技术等快速发展的一年。毫无疑问, 我们已生 活在一个成千上万人正在发现和发明一系列令人眼花 缭乱的令人兴奋的科学技术时代, 我们将继续站在 巨人的肩膀，为迎接新的一年憧憬着并做好准备。 